\title{
Localized Component Analysis for Arthritis Detection in the Trapeziometacarpal Joint
}

\author{
Martijn van de Giessen ${ }^{1,2,5}$, Sepp de Raedt ${ }^{1, \star}$, Maiken Stilling ${ }^{3}$, \\ Torben B. Hansen ${ }^{3}$, Mario Maas ${ }^{4}$, Geert J. Streekstra ${ }^{5}$, \\ Lucas J. van Vliet ${ }^{1}$, and Frans M. Vos ${ }^{1,4}$
}

1 Quantititative Imaging Group, Delft University of Technology, The Netherlands

2 Division of Image Processing, Leiden University Medical Center, The Netherlands

3 Dept. of Orthopaedics, Holstebro Regional Hospital, Denmark

4 Dept. of Radiology, AMC Amsterdam, The Netherlands

5 Dept. of Biomed. Engineering and Physics, AMC Amsterdam, The Netherlands

\begin{abstract}
The trapeziometacarpal joint enables the prehensile function of the thumb. Unfortunately, this joint is vulnerable to osteoarthritis (OA) that typically affects the local shape of the trapezium. A novel, local statistical shape model is defined that employs a differentiable locality measure based on the weighted variance of point coordinates per mode. The simplicity of the function and the smooth derivative enable to quickly determine localized components for densely sampled surfaces.

The method is employed to assess a set of 60 trapezia (38 healthy, 22 with OA). The localized components predominantly model regions affected by OA, contrary to shape variations found with PCA. Furthermore, identification of pathological trapezia based on the localized modes of variation is improved compared to PCA.
\end{abstract}

\section{Introduction}

The prehensile biomechanical function of the thumb is determined for a large part by the complex saddle-shaped trapeziometacarpal (TMC) joint (Fig. 1a). Unfortunately, the unique motion of the TMC joint renders trapezium and metacarpal 1 vulnerable to impairment by osteoarthritis (OA), e.g. 7]. Depending on the progression of the disease, the symptoms range from discomfort to the complete inability to manipulate objects. Initially, OA manifests as local, destructive shape changes of the adjacent joint surfaces [4] that result in joint space narrowings and affect motion patterns. Such shape changes are particularly difficult to diagnose using conventional X-ray images. We hypothesize that a model of known shape variations can aid in detecting pathological changes.

A common approach to describe shape variations is to use active shape models (ASM) 3]. An ASM models coordinate distributions of surface points on example shapes by a mean shape and orthogonal modes of variation using principal component analysis (PCA). However, PCA typically describes global shape

\footnotetext{
* M. van de Giessen and S. de Raedt contributed equally to this work.
} 
variations. It has been observed, mainly in segmentation applications, that these global descriptions are not always appropriate to model local variations in shape, e.g. 8]. A well-known alternative to PCA is independent component analyis (ICA). Although ICA tends to describe shape variations in a more localized way [10, the ICA components are non-orthogonal. Since a shape representation using non-orthogonal components is not unique, this complicates a statistical comparison between healthy and pathological shapes.

Two methods that do preserve the orthogonality between components are VARIMAX [6] and sparse component analysis (SPCA) as proposed in [2]. Both techniques aim to minimize the number of point coordinates that are affected by each mode. However, there is not an explicit optimization for spatially coherent variations. To improve on this aspect, Alcantara et al. 1 proposed a method for localized component analysis (LoCA) in hippocampi. This method adapts the cost function from [2] with a term that penalizes concurrent movement of distant surface points. A limitation, though, is that the proposed localization term in [1] does not have a continuous derivative. This makes the method difficult to optimize using gradient descent methods and thereby sensitive to local minima.

In this work we propose a new method for localized component analysis with a penalty function for non-locality that has continuous 1st and higher order derivatives. Using this function a localized statistical model is defined for a set of 38 healthy and 22 pathological trapezia. The local shape variations are used in the design of a classifier for identification of pathological trapezia.

\section{Materials and Methods}

The method proposed in this work uses a point distribution model (PDM) as used in ASMs [3]. However, instead of using the principal components of variation, determined by PCA, the components are optimized to explicitly model local variations. Therefore a novel, differentiable locality measure is derived.

\subsection{Active Shape Models}

A PDM assumes a set of example shapes, represented by points for which the correspondence relations to a point on any of the other shapes have been established. It comprises a linear model that describes how the surface points $\mathbf{x}_{i}$ of shape $S_{i}$ deviate from the mean $\overline{\mathbf{x}}$ of a set of example shapes:

$$
\mathbf{x}_{i}=\overline{\mathbf{x}}+P \mathbf{b}_{i}
$$

where $\mathbf{x}_{i}=\left[\begin{array}{lllllll}x_{1} & y_{1} & z_{1} & \cdots & x_{N} & y_{N} & z_{N}\end{array}\right]^{T}$ contains the concatenated coordinates of $N$ points on surface $S_{i}, \overline{\mathbf{x}}$ describes the mean shape, $P=\left[\mathbf{p}_{1} \mathbf{p}_{2} \cdots \mathbf{p}_{M}\right]$ contains the $M$ modes of variation as columns $\mathbf{p}_{j}, j=1, \ldots, M$ and $\mathbf{b}_{i}$ is a vector with weightings for each mode. When the columns of $P$ are orthogonal, $\mathbf{b}_{i}$ uniquely describes surface $S_{i}$.

Thus, a set of surfaces is described with the mean coordinate vector $\overline{\mathbf{x}}$ and a matrix $B$ where each column $\mathbf{b}_{i}$ describes a different example surface. The 


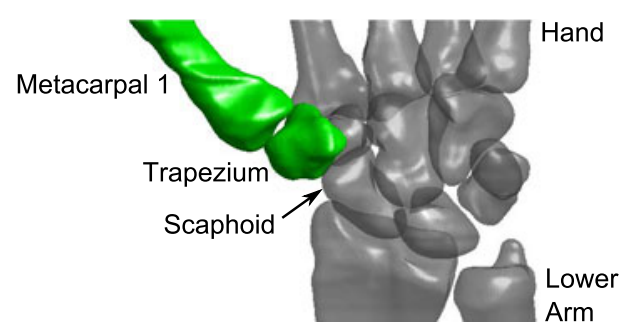

(a)

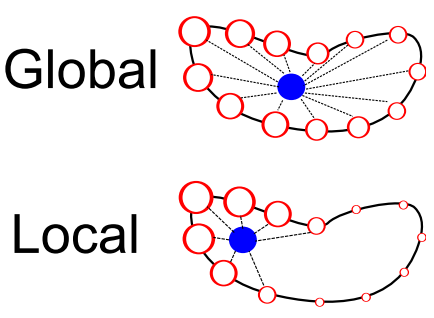

(b)

Fig. 1. (a) The TMC joint in a left-handed wrist. (b) Schematic overview of the weighted variance as a measure for locality. The radius of the red circles indicates the magnitude of the variation of the surfaces points in a mode. The blue dots are the weighted means $\mathbf{m}_{j}$. The sum of the lengths of the dashed lines squared, weighted with the squared magnitudes of the variations is the localization measure in (6).

variance per mode $\sigma_{j}^{2}$ (for mode $j$ ) is a measure for the 'importance' of a mode and is estimated as the variance of each row of $B$. It is customary to compute the relative variance per mode as $v_{j}=\sigma_{j}^{2} / \sum_{k=1}^{M} \sigma_{k}^{2}$. PCA is often implemented using an eigenvalue or singular value decomposition, but the same $P$ can also be obtained by rotating the components and thereby minimizing [2]:

$$
C_{\text {pca }}(P)=\sum_{j=1}^{M}-v_{j} \log \left(v_{j}\right)
$$

A common strategy for such a minimization is to pairwise rotate components in the plane spanned by them until $C_{\text {pca }}$ is minimal [2]1. This procedure ensures that the rotating components remain orthogonal to all other components. The formulation (2) has the advantage that it can be extended to sparse or local component analysis, where a trade-off between compactness of the model (PCA) and other criteria is needed such as sparsity or locality.

\subsection{Localized Component Analysis}

For a localized description of shape variation within a set the following cost function is minimized (similar to [2]):

$$
C(P)=(1-\lambda) C_{\text {pca }}(P)+\lambda C_{\text {loca }}(P)
$$

where $\lambda$ is a parameter to balance between compactness $\left(C_{\text {pca }}\right)$ and locality $\left(C_{\text {loca }}\right)$. The latter will be derived in this section.

Each column $\mathbf{p}_{j}$ of $P$ is normalized $\left(\left\|\mathbf{p}_{j}\right\|=1\right)$ and contains the relative displacements of all surface points compared to $\overline{\mathbf{x}}$. The relative displacement per surface point (in $3 \mathrm{D}$ ) is thus given by each triplet of elements of vector $\mathbf{p}_{j}$ :

$$
\mathbf{p}_{j}=\left[\mathbf{d}_{j, 1} ; \cdots ; \mathbf{d}_{j, N}\right]
$$


Here $\mathbf{d}_{j, k}$ is the 3-element vector with the displacement of surface point $k$. The relative displacement amplitudes of all points are thus given by $d_{j, k}=$ $\left\|\mathbf{d}_{j, k}\right\|$. The newly derived locality measure in this work uses these displacement measures as weights in a variance analysis of the coordinates of the affected points in the following manner.

Intuitively, one might observe that points with large relative displacements should be close together in a description of local variations (Fig. (1b)). In other words, the distribution of points that have a high value of $d_{j, k}$ should be compact. Therefore, a measure for the compactness of large variations is the variance of the coordinates weighted by $d_{j, k}$. The most likely estimate of the set of varying shapes is given by the mean shape and therefore the weighted variance is computed on the mean cloud $\overline{\mathbf{x}}$. By denoting the coordinate vector for each point in $\overline{\mathbf{x}}$ as $\mathbf{z}_{k}=\left[\begin{array}{lll}x_{k} & y_{k} & z_{k}\end{array}\right]^{T}$, the weighted variance vector for the $j$-th mode is given by

$$
\mathbf{s}_{j}=\sum_{k=1}^{n} d_{j, k}^{2}\left(\mathbf{z}_{k}-\mathbf{m}_{j}\right)^{2} \text { where } \mathbf{m}_{j}=\sum_{k=1}^{N} d_{j, k}^{2} \mathbf{z}_{k}
$$

is the weighted mean point. The weighted mean $\mathbf{m}_{i}$ can be interpreted as the point around which the displacement concentrates. Note that this point does not have to be positioned on the shape surface. The cost function $C_{\text {loca }}$ is then composed from the weighted variance vectors as

$$
C_{\text {loca }}(P)=\sum_{j=1}^{M} \sum_{l=1}^{d} s_{j, l}
$$

where $d$ is the number of dimensions and $s_{j, l}$ is the $l$-th element of $\mathbf{s}_{j}$. We dub this method Localized Variance Component Analysis (LVCA).

To obtain localized components, $C(P)$ is minimized using sequential pairwise rotations of components as in 2]. This split in pairwise rotations is allowed because both $C_{\text {pca }}(P)$ and $C_{\text {loca }}(P)$ are summations in which the terms that depend on non-rotated components remain constant. A crucial aspect of our method is that (5) is by definition a smooth function that has a continuous derivative. Therefore (3) can be minimized efficiently with a gradient-descent minimization. We employ the Boyden-Fletcher-Goldfarb-Shanno algorithm.

\section{$3 \quad$ Experiments}

The advantages of a localized statistical shape model over a PCA model are demonstrated in two datasets. Initially, the effect of the parameter $\lambda$ is studied in an artificial set of facial contours. Subsequently, the shape changes between healthy trapezia and trapezia with OA are assessed and used for classification.

\subsection{Data}

Faces. The 'faces dataset' was obtained from the IMM Face Database [9] that consists of 240 images of faces annotated with 58 points each. The focus was on the 120 full frontal faces (the others presenting lateral views). Translations, rotations and scale differences were removed with a Procrustes analysis. 
Trapezia. Two datasets of CT scans were separately acquired: a set of healthy trapezia and a set with trapezia affected by osteoarthritis.

The normal dataset contains 38 CT scans with a mean age of 51 (21-70) years. All CT scans were obtained as a result of bilateral scanning over a period of two years at the Academic Medical Center, The Netherlands. Individuals in this set had no complaints (pain, dysfunction) regarding included wrists and all wrist bones were fully developed (epiphyseal lines closed). An experienced musculoskeletal radiologist (MM) deemed all included wrists as healthy and excluded any wrists showing signs of: fractures, arthritis, fragments and fusions.

The OA group contained 22 CT scans from patients with a mean age of 58 (4072 ) years. The CT scans were consecutively acquired over a period of one year from Holstebro Regional Hospital and Herning Regional Hospital, Denmark. The dataset contained preoperative scans from patients who received a TMC joint prosthesis. The patients had all been referred to the department of hand surgery due to thumb pain and dysfunction. They were all graded for TMC OA using the Eaton Glickel Classication system by an experienced hand surgeon (TBH) using the anteroposterior (AP) radiographs [4. The group was comprised of 6 stage II and 18 stage III patients with TMC OA in addition to a single patient with stage I and a single patient with stage IV TMC OA.

All scans were resampled to have an isotropic voxel size of $0.3 \times 0.3 \times 0.3 \mathrm{~mm}^{3}$. The voxel sizes in the original scans slightly varied, but were almost isotropic with voxel volume differences in the order of $10 \%$. The trapezia in both sets were segmented using a level set based method, with the same parameter settings as in [5] $10^{4}$ points per bone surface, corresponding between the bones, were established using non-rigid registration similar to [5].

\section{$3.2 \quad$ Faces}

The modes of variations determined by the proposed localized component analysis method (LVCA) were compared to those found by PCA and the method proposed by Alcantara [1] (LoCA). Alcantara's method needs a user-defined function that describes the locality. Therefore, we chose a non-normalized Gaussian: $\exp \left(-q^{2} / 2 \sigma^{2}\right)$, with $\sigma=0.02$, where $q$ is the distance between points and the face coordinates are between 0 and 1 in horizontal and vertical direction. For both LoCA and LVCA, the influence of the parameter $\lambda$ was studied.

The 10 modes of variation with the largest variance per mode are shown in Fig. 2. The PCA model (effectively $\lambda=0$ in (3) ) clearly distinguishes itself from the other models by the global variations. For the other extreme case $(\lambda=1)$, both LoCA and LVCA render variations that are too sparse, which is observable as several modes only perturb a single point. Clearly, such extremely localized components are not very informative. This behavior arises because the dataset contains more shapes $(120)$ than point coordinates $(2 \times 58=116)$. Reversely, if there are less example shapes than point coordinates, then by definition any mode of variation must affect more than one point. Setting $\lambda<1$ allows for a trade-off between local and principal components. This can be seen in the rows 


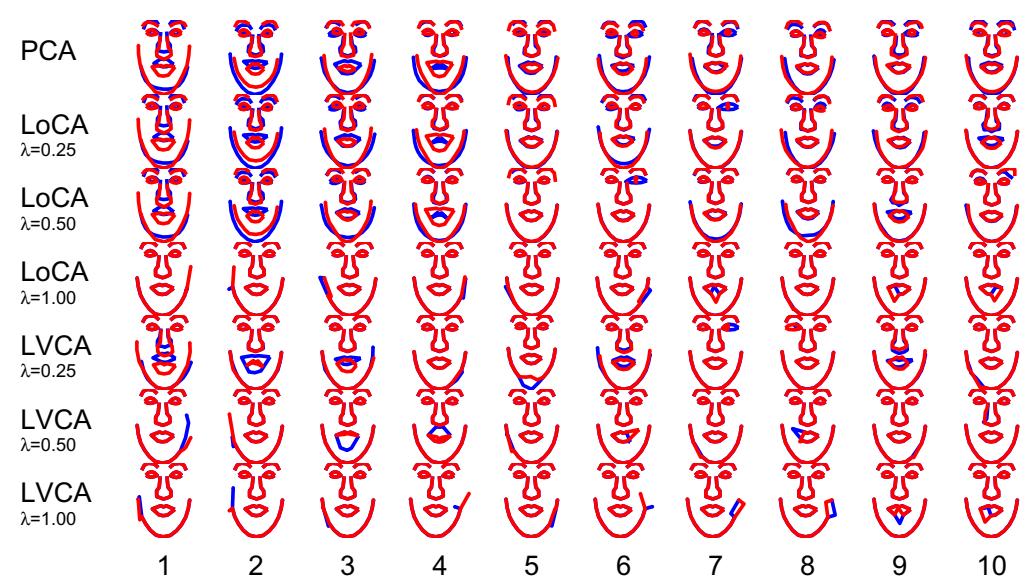

Fig. 2. The 10 modes of variation with the highest variance in the IMM faces data for PCA, localized components as in 1] (LoCA) and as proposed (LVCA), both for $\lambda=1,0.5,0.25$. For each mode the deviations from the mean shape at $+/-3$ standard deviations are shown.

with $\lambda=0.25,0.5$ for both LoCA and LVCA of Fig. 2, which show variations that are locally concentrated (e.g. around the mouth, nose or eyes), but leave structures further away unaffected. However, LoCA easily gets stuck in local minima, which is demonstrated in modes 1 to 4 for $\lambda=0.25,0.5$, which are (almost) the same as the corresponding PCA modes. Computing components was approximately 130 times faster for LVCA than for LoCA.

\subsection{Trapezia}

The trapezia experiment compared PCA and the proposed LVCA to identify shape changes between healthy and pathological shapes. Clearly, the number of point coordinates $\left(3 \times 10^{4}\right)$ is vastly larger than the number of example trapezia (60). To obtain the most localized components possible in this set, $\lambda$ in (3) was set to 1 . The coefficients of the modes of variations for the PCA and LVCA model are denoted as $\mathbf{b}_{i}^{\mathrm{PCA}}$ and $\mathbf{b}_{i}^{\mathrm{LVCA}}$, respectively. Using the coefficients with the largest discrepancy between healthy and pathological bones, a linear logistic classifier is trained. The number of features used is optimized using forward feature selection. The performance of feature selection and classifier training were tested by a 50 times repeated cross validation.

Fig. 3a demonstrates that for PCA the coefficients of the healthy and pathological trapezia lie (in general) closer together than for LVCA. This is to be expected, as the changes of the bone surface due to osteoarthritis are mainly local. In Fig. 4 the 10 modes with the largest differences between healthy and pathological coefficients are shown, in which the color reflects the values $d_{j, k} \times \sigma_{j}$ (See (4)), i.e. the average deviation (in mm). Clearly, the modes of the PCA 


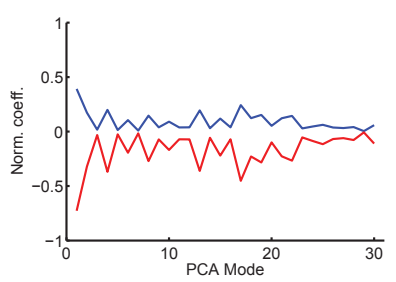

(a)

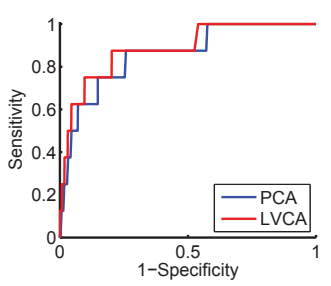

(b)

Fig. 3. (a) Normalized means of $\mathbf{b}_{i}^{\mathrm{PCA}}$ and $\mathbf{b}_{i}^{\mathrm{LVCA}}$ (i.e. expressed in terms of the corresponding standard deviations (SD)) of the first 30 modes for PCA (left) and LVCA (right), for healthy (blue,up) and pathological shapes (red,down). Split healthy and pathological SDs are just below 1. (b) Average ROC curves (over 50 experiments) for a logistic linear classifier using $\mathbf{b}_{i}^{\mathrm{PCA}}$ and $\mathbf{b}_{i}^{\mathrm{LVCA}}$ as features.

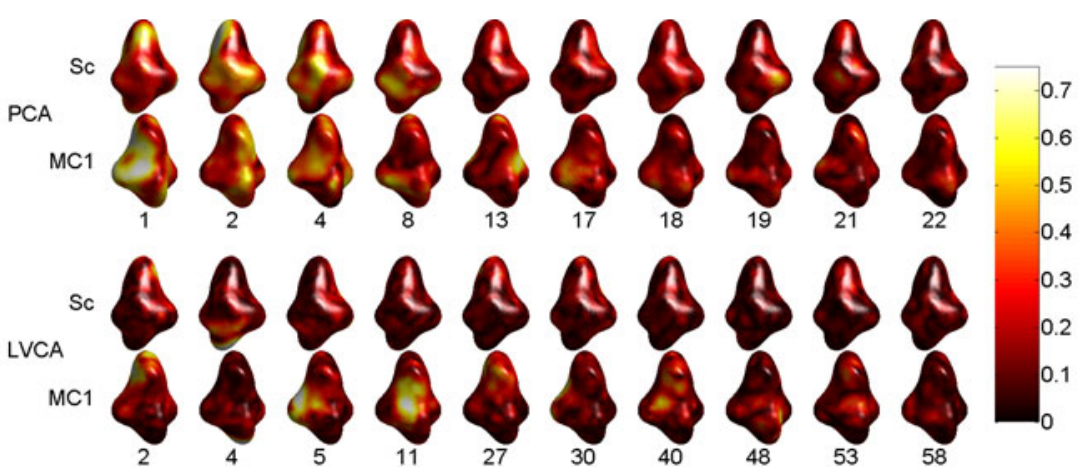

Fig. 4. The 10 modes of variation, sorted by decreasing variance, for which OA trapezia differ the most from healthy trapezia, for PCA and LVCA. Both sides of the trapezium adjacent to the scaphoid (Sc) and metacarpal 1 (MC1) are shown. Intensity increases with variation amplitude (in $\mathrm{mm}$ ).

model affect the shape in a more global way than the local modes. Particularly the local modes 2, 4 (at the bottom edge), 27 and 30 show the locations of osteophytes, whereas modes 5 and 11 show the flattening of the surface of the TMC joint. The four last local modes in Fig. 4 (40, 48, 53, 58) contain very small variations, which are mainly noise. Observe that the global characteristics of the PCA modes make that they cannot be related to specific bone surface locations that are affected by osteoarthritis. The average receiver operating curves (ROC) for the classifiers are shown in Fig. $3 \mathrm{~b}$. These curves show that the LVCA components give a slightly better trade-off between sensitivity and specificity than the PCA components. We hypothesize that this must be attributed to the higher sensitivity of LVCA to local deviations as the classification improvement mainly concerns trapezia with small osteophytes and that deviate as in LVCA mode 11 . 


\section{Discussion}

We proposed a new method for efficient modeling of local shape variations. A novel, differentiable locality measure was defined, which effectively equals a $3 \mathrm{D}$ weighted variance of the point coordinates per mode. The simplicity of the function and the smooth derivative enable to quickly determine localized components for densely sampled surfaces. The LVCA models based on the IMM faces dataset clearly show that the locality of the modes depends on the trade-off parameter $\lambda$. For (relatively) small sets of densely sampled shapes, such as the trapezia, the number of components in the trainingset is sufficiently limited to obtain clinically meaningful local shape variations for $\lambda=1$. Larger, sparser training sets require a $\lambda<1$. Estimating LVCA components was shown to be less sensitive to local minima and 2 orders of magnitude faster than LoCA components in the IMM faces set. The LVCA modes corresponded to trapezoid surfaces variations where changes are expected from clinical practice. Reversely, for PCA components the variations were global. Particularly, the area around the facet adjacent to the scaphoid bone was (incorrectly) detected to contain important variations. Classification of pathological trapezia showed a consistent improvement with LVCA coefficient features, compared to PCA.

\section{References}

1. Alcantara, D.A., Carmichael, O., Harcourt-Smith, W., Sterner, K., Frost, S.R., Dutton, R., Thompson, P., Delson, E., Amenta, N.: Exploration of shape variation using localized components analysis. IEEE T. Pattern Anal. 31(8), 1510-1516 (2009)

2. Chennubhotla, C., Jepson, A.: Sparse PCA - Extracting multi-scale structure from data. In: ICCV 2001, vol. 1, pp. 641-647 (2001)

3. Cootes, T.F., Taylor, C.J., Cooper, D.J., Graham, J.: Active shape models - their training and application. Comput. Vis. Image Und. 61(1), 38-59 (1995)

4. Eaton, R.G., Glickel, S.Z.: Trapeziometacarpal osteoarthritis. staging as a rationale for treatment. Hand Clin. 3(4), 455-471 (1987)

5. Van de Giessen, M., Foumani, M., Streekstra, G.J., Strackee, S.D., Maas, M., van Vliet, L.J., Grimbergen, C.A., Vos, F.M.: Statistical descriptions of scaphoid and lunate bone shapes. J. Biomech. 43(8), 1463-1469 (2010)

6. Kaiser, H.: The VARIMAX criterion for analytic rotation in factor analysis. Psychometrika 23(3), 187-200 (1958)

7. North, E.R., Rutledge, W.M.: The trapezium-thumb metacarpal joint: the relationship of joint shape and degenerative joint disease. Hand 15(2), 201-206 (1983)

8. Shen, D., Herskovits, E.H., Davatzikos, C.: An adaptive-focus statistical shape model for segmentation and shape modeling of 3-D brain structures. IEEE T. Med. Imaging 20(4), 257-270 (2001)

9. Stegmann, M.B., Ersbøll, B.K., Larsen, R.: FAME - a flexible appearance modelling environment. IEEE Trans. Med. Imaging 22(10), 1319-1331 (2003)

10. Suinesiaputra, A., Frangi, A.F., Kaandorp, T.A., Lamb, H.J., Bax, J.J., Reiber, J.H.C., Lelieveldt, B.P.F.: Automated detection of regional wall motion abnormalities based on a statistical model applied to multislice short-axis cardiac MR images. IEEE T. Med. Imaging 28(4), 595-607 (2009) 